\title{
Persistent Primitive Olfactory Artery Aneurysm -Case Report-
}

\author{
Tetsuya YAmAmoto, Kensuke SUZUKI, Tomosato YAMAZAKI, \\ Wataro TSURUTA, Takao TSURUBUCHI, and Akira MATSUMURA \\ Department of Neurosurgery, Graduate School of Comprehensive Human Sciences, \\ University of Tsukuba, Tsukuba, Ibaraki
}

\begin{abstract}
A hypertensive 69-year-old man presented with an incidentally discovered non-ruptured aneurysm of the persistent primitive olfactory artery (POA). The POA originates at the terminal portion of the internal carotid artery, runs along the olfactory tract anteriorly toward the crista galli, and forms an acute angle with the distal anterior cerebral artery. The aneurysm was located at this characteristic acute angle. The persistent POA aneurysm associated with an anterior communicating artery aneurysm with wide neck was successfully treated by clipping surgery.
\end{abstract}

Key words: persistent primitive artery, aneurysm

\section{Introduction}

Cerebral artery aneurysms may occur in association with embryonic carotid-basilar anastomoses such as primitive trigeminal artery and primitive hypoglossal artery, ${ }^{1,7)}$ as well as anomalies associated with the anterior cerebral artery (ACA), including azygos ACA, fenestration, superior anterior communicating artery (AComA), anomalous branch of ACA, interoptic course of ACA, and carotid-ACA anastomosis. ${ }^{8,9)}$ Here we report a rare case of non-ruptured aneurysm associated with persistent primitive olfactory artery (POA), which is another rare anomaly of the ACA.

\section{Case Report}

A hypertensive 69-year-old Japanese man who was incidentally diagnosed with non-ruptured cerebral artery aneurysms of the anomalous ACA branches when he experienced loss of consciousness in the setting of alcoholism. A review of his family history indicated that his sister had suffered subarachnoid hemorrhage. His hypertension had been well controlled with nifedipine for more than 10 years. He had never experienced headache, anosmia, or visual disturbance.

Neurological examination on admission revealed no deficit. Computed tomography of the head

Received July 10, 2008; Accepted January 30, 2009 showed no abnormalities. Three-dimensional computed tomography angiogram and digital subtraction angiography revealed an anomalous artery arising from the terminal portion of the right internal carotid artery (ICA) (Figs. 1 and 2). The proximal portion of the anomalous artery ran anteromedially and made a hair-pin turn posterosuperiorly, associated with a non-ruptured saccular aneurysm 13.3 $\mathrm{mm}$ in diameter without branches originating around the aneurysm. The anomalous artery was in-
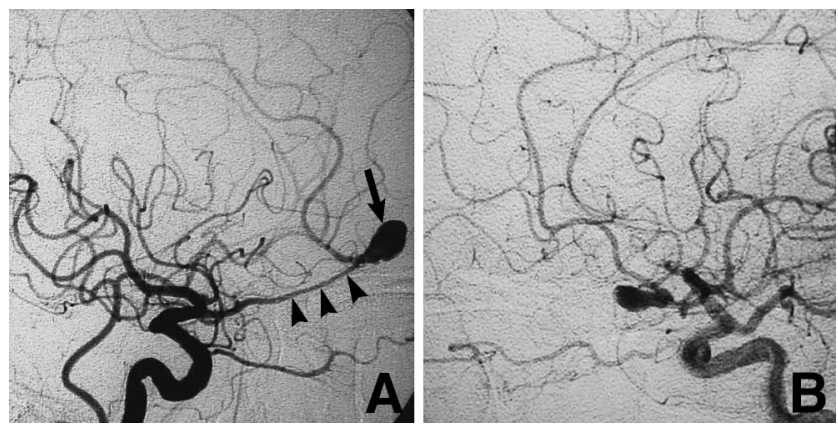

Fig. 1 Right (A) and left (B) internal carotid angiograms, lateral views, showing a saccular aneurysm (A, arrow) arising from the distal end of the primitive persistent olfactory artery (A, arrowheads), which makes an acute angle with the distal anterior cerebral artery, and an associated aneurysm arising from the $A_{1}-A_{2}$ junction (B). 


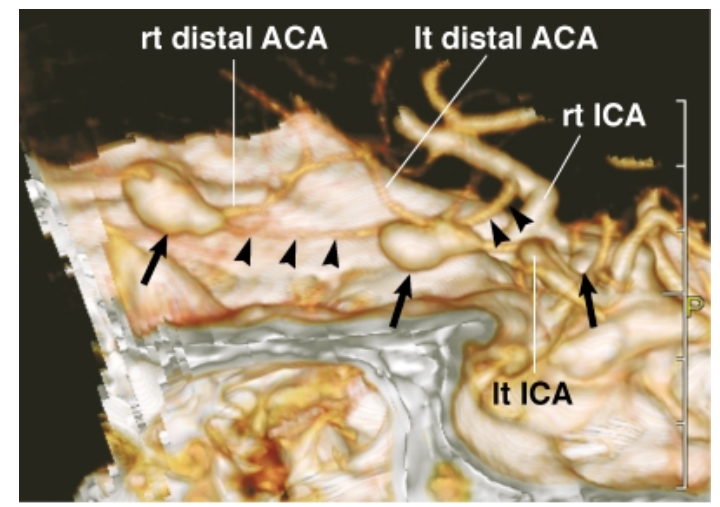

Fig. 2 Three-dimensional computed tomography angiogram showing the anatomical relationships among the bilateral internal carotid arteries (ICAs), right persistent primitive olfactory artery (arrowheads), right distal anterior cerebral artery (ACA), left distal ACA, and aneurysms (arrows) of the persistent olfactory artery, anterior communicating artery, and right ICA.

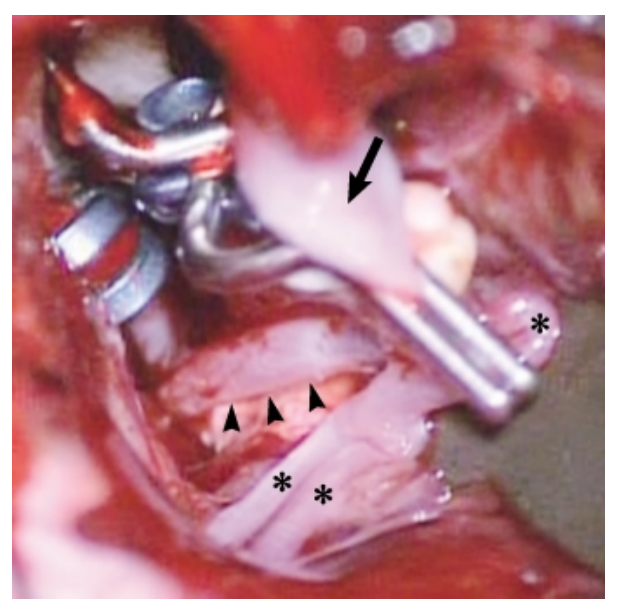

Fig. 3 Intraoperative photograph showing the anatomical relationship among the right persistent primitive olfactory artery (arrowheads), clipped aneurysm of the persistent olfactory artery (arrow), and distal anterior cerebral artery and its branches (asterisks).

terpreted as a persistent POA. The distal ACA coursed posteriorly, like a normal distal ACA. Angiography detected no delineation of the AComA nor cross-filling via the AComA. Non-ruptured aneurysms were found of $9.2 \mathrm{~mm}$ in diameter at the left $A_{1}-A_{2}$ junction and $3.3 \mathrm{~mm}$ in diameter on the ICA-ophthalmic portion.

Surgical intervention was performed to prevent further rupture of the two relatively large aneurysms at the persistent POA and the left $\mathrm{A}_{1}-\mathrm{A}_{2}$ junction. Clipping was performed through an interhemispheric approach rather than coil embolization because of accessibility to the distal ACA and the broad aneurysm neck (Fig. 3). The aneurysms were completely clipped and the patient's postoperative course was uneventful.

\section{Discussion}

The POA originates as the distal derivative of the ICA at 5 weeks gestation, which transiently supplies the nasal cavity along the olfactory tract, and its predominant branch normally persists only as the recurrent artery of Heubner. ${ }^{6)}$ Typically, the persistent POA originates at the terminal portion of the ICA, runs along the olfactory tract anteriorly toward the crista galli, and the distal portion of the POA makes an acute angle with the distal ACA, which supplies the normal ACA territory. Fifteen cases of POA including ours have been reported..$^{2-5,8-11)}$ Twelve cases occurred unilaterally, and 7 were associated with ruptured or non-ruptured aneurysm, and usually showed agenesis of the ipsilateral recurrent artery of Heubner.

The neck of the aneurysm is usually located at the characteristic hair-pin turn of the persistent POA, so the aneurysms are thought to be caused by congenital frailness or changes in hemodynamics. ${ }^{9}$ All 7 patients with aneurysms including the 3 patients with ruptured aneurysms underwent craniotomy. In the present case, we considered that the persistent POA aneurysm associated with an AComA aneurysm with wide neck would be better treated by clipping surgery. Coil embolization would be possible for a persistent POA aneurysm with a narrow neck and relatively better accessibility to catheterization.

\section{References}

1) Baltsavias GM, Chourmouzi D, Tasianas N, Drevelengas A, Damianovski D, Jovkovski S: Ruptured aneurysm of a persistent primitive hypoglossal artery treated by endovascular approach - case report and literature review. Surg Neurol 68: 338-343, 2007

2) Enomoto H, Goto H, Murase M: [A ruptured aneurysm of the frontoorbital artery and the coexisting anastomosis with the anterior ethmoidal artery]. No Shinkei Geka 14: 203-206, 1986 (Jpn, with Eng abstract)

3) Hanakita L, Nagayasu S, Nishi S, Suzuki T: [An aneurysm of the distal anterior cerebral artery with a remarkably anomalous configuration]. No Shinkei Geka 16: 781-784, 1988 (Jpn, with Eng abstract) 
4) Kaneko T, Suetake K, Shinya T, Takeda M: [Persistent primitive olfactory artery: report of 5 cases]. No Shinkei Geka 27: 933-939, 1999 (Jpn, with Eng abstract)

5) Moffat DB: A case of persistence of the primitive olfactory artery. Anat Anz 121: 477-479, 1967

6) Nozaki K, Taki W, Kawakami O, Hashimoto N: Cerebral aneurysm associated with persistent primitive olfactory artery aneurysm. Acta Neurochir (Wien) 140: 397-402, 1998

7) Onizuka M, Kazekawa K, Tsutsumi M, Kodama T, Aikawa H, Ikou M, Tomokiyo M, Matsubara S, Nii K, Tanaka A: Hyperform remodeling balloon for the balloon occlusion test of persistent primitive trigeminal artery aneurysm - case report. Neurol Med Chir (Tokyo) 46: 541-543, 2006

8) Takeshita G, Katada K, Koga S, Sano H, Kanno T: [An anomalous course of proximal portion of the anterior cerebral artery]. Rinsho Hoshasen 33: 593-596, 1988
(Jpn)

9) Tsuji T, Abe M, Tabuchi K: Aneurysm of a persistent primitive olfactory artery. Case report. J Neurosurg 83: 138-140, 1995

10) Uchino A, Sawada A, Takase Y, Kudo S: Persistent primitive olfactory artery: Diagnosis with MR angiography. J Clin Imaging 25: 258-261, 2001

11) Yamaura A, Takase M, Makino H: An aneurysm of the proximal anterior cerebral artery at the olfactory bulb. Surg Neurol 12: 425-427, 1979

Address reprint requests to: Tetsuya Yamamoto, M.D., Department of Neurosurgery, Graduate School of Comprehensive Human Sciences, University of Tsukuba, 1-1-1 Tennodai, Tsukuba, Ibaraki 305-8575, Japan. 